\title{
Connecting the dots between mental and menstrual health
}

Author: Esther Ejiroghene Ajari

\section{Affiliation:}

1. Faculty of Clinical Sciences, College of Medicine, University of Ibadan, Ibadan, Oyo State, Nigeria

2. The TriHealthon, Ibadan, Oyo State, Nigeria

Physical Address: C1, Alexander Brown Hall, University College Hospital, Ibadan, Oyo State, Nigeria

Email Address: estherejiroghene@gmail.com

Phone Number: +2347017011997

\begin{abstract}
Context:

Mental and menstrual health, two emerging aspects of health critical to the female population, are bidirectionally linked. But there is limited empirical evidence that establishes the association between these entities. This essay aims to draw the attention of researchers to this healthcare niche.
\end{abstract}

\section{Evidence Acquisition:}

A systematic review was carried out on literatures sourced from medical databases (e.g. PubMed, Google Scholar, Scopus, Science Direct, and Web of Science), and gray sources - popular and reputable institutional and journalistic websites that publishes mental or menstrual health research.

The key words used for the search are "Menstruation, menstrual hygiene, menstrual disorders, premenstrual syndrome, period poverty, menarche, menopause, mental health, mental disorder, mental illness, depression, anxiety, phobia, mania, mood, and affect".

The initial search generated 368 results. But after the duplicates were removed, the exclusion criteria (publication before 2000) was applied, and manual review of abstract (for relevance) was done, 21 publications from the databases and 5 from gray sources were included in this essay.

\section{Results:}

Associations were found between menstrual cycle irregularities and disorders; and mental disorders such as premenstrual syndrome, premenstrual dysphoric disorder, major depressive disorder, major anxiety disorder, psychotic disorder, bipolar disorder, borderline personality disorder, sleep disorder, substance use/abuse, and suicidal ideation and attempts.

Few positive associations between mental and menstrual health were recorded, and several research and treatment gaps were identified.

\section{Conclusion:}


Research into the links between mental and menstrual health should not remain a fringe area of scientific curiosity, as it shows tremendous promise in improving healthcare offered to women/girls globally.

Keywords: Menstruation, menstrual health, menstrual hygiene, period poverty, mental health, mental disorder

\section{Context}

Current societal assumptions about mental health and menstruation have generally been negative. Apart from the common-shared widespread misunderstanding about them, these entities are intricately linked in the biopsychosocial domain as well. There are strong empirical evidences, albeit limited, that demonstrates that the course of one's mental health can be influenced by one's menstrual health, and vice versa [1]. Menstrual health, here, refers to the health and wellbeing of women and girls (jointly referred to as "women" throughout the rest of this paper) during the follicular, menstrual and luteal phases of their menstrual cycle (MC).

The associations between mental and menstrual health can be both positive and negative. The negative associations can come either in the form of pre-menstrual and peri-menstrual exacerbation of existing mental disorders, or onset of new mental illness symptoms or syndrome. This is similar to the way MC, sometimes, influences physical well-being_ovarian disorders, pregnancy, migraines, cardiovascular diseases etc. [1].

Menstrual-related mental disorders (MRMD), which is highly prevalent among young women, are a group of clinically viable affective, behavioral, and physical symptoms as well as functional disorders, occurring in the late luteal MC phase and usually resolving few days after the onset of menstruation [1].

The disability adjusted life years lost due to an MRMD is almost the same as those lost due to major mental disorders [2]. However, research into MRMD remains a fringe area of scientific curiosity globally, even among the most-funded psychiatry research departments. Moreover, most of the studies into this subject matter have focused on the Caucasian populations alone, and as such, there is very few evidences that the reported results are applicable to women of other races.

These are the reasons why this paper systematically and comprehensively reviews the reported positive and negative associations between mental and menstrual health; the empirical body of evidence that suggests the reasons for these associations; and the research and treatment gaps that exist in this discourse.

\section{Evidence Acquisition:}

\section{Search Strategy}

This systematic review, of quantitative and qualitative data, was carried out on literatures sourced from medical databases such as PubMed, Google Scholar, Web of Science, Science Direct, and Scopus. Given the comparatively limited amount of supporting evidence on this subject matter, gray sources (such as popular and reputable institutional and journalistic websites that publishes mental or menstrual health research) were also consulted. 
The key words used for the search are "Menstruation, menstrual hygiene, menstrual disorders, premenstrual syndrome, period poverty, menarche, menopause, mental health, mental disorder, mental illness, depression, anxiety, phobia, mania, mood, and affect".

\section{Inclusion and Exclusion Criteria}

The initial literature search generated 368 results. After duplicate articles were removed, the result came down to 169 articles. After the exclusion criteria (publication before 2000) was applied, only 99 articles were left.

Thereafter, to exclude superfluous articles not relevant to the study, manual review of abstracts and titles was done. This process yielded 21 publications from the databases and 5 from gray sources, which were then included in this review.

\section{Results}

\section{Menstrual Cycle (MC) Irregularities}

Studies have shown that in some women, the occurrence of MC irregularities increases with the number of existing mental disorders, especially those involving low mood [3]. Also, psychological counseling, and high stress levels (associated with mental disorders) have been shown to increase the risks of MC irregularities among women, after adjusting for cofounding factors such as age, body mass index, substance use/abuse status, physical activity, age at menarche, hemoglobin level, household income, weight loss attempts, and sleep regularity and duration [3].

Furthermore, studies have shown that women who experienced dysmenorrhea, and longer, irregular and heavy periods had significantly lower psycho-social functioning, poorer general health perceptions, and are more constrained by societal role limitations than those without these irregularities [4]. Also, in Caucasian women, those with shorter cycles ( $\leq 28$ days) have 1.5-2.0 times more risk of current and lifetime affective disorders [1].

\section{Premenstrual Syndrome (PMS)}

The most common mental disorder associated with the MC is PMS [4], which according to the Diagnostic and Statistical Manual of Mental Disorders, is a type of depressive disorder, characterized by a group of recurrent, moderate, non-psychotic psychological and physical symptoms that presents during the luteal MC phase and resolve during the menstrual phase.

More than 200 of PMS symptoms have been identified over the last fifty years, and these are classified into three main domains: emotional, behavioral and physical. The most commonly reported ones include psychological symptoms (such as anger, irritability, decreased concentration, restlessness, confusion or mood swings); distressing behaviors (such as fatigue, reduced libido, insomnia etc.) [4]; and physical symptoms (such as breast swelling and tenderness [5], headaches, back pain, abdominal bloating, and muscle or joint pain [4]).

Mild PMS that does not affect social functioning occurs in approximately $75 \%$ of pre-menopausal women, while moderate and severe PMS that affects social functioning occurs in 20-32\% of these women [4].

An epidemiological study established that the prevalence of concurrent major depressive and anxiety disorders and the risk of future occurrence of incidental affective disorders is much higher in women who have suffered from PMS than in those who have not [5]. 
Additionally, in 2019, Matsumoto et al. reported a higher prevalence of severe or extremely severe psychosocial symptoms among women who consider themselves "unhealthy and stressed" during their MC than among those who consider themselves "healthy and non-stressed" [6].

\section{Premenstrual Dysphoric Disorder (PMDD)}

PMDD, which is different from PMS primarily in the number, duration, severity and quality of symptoms, affects 3\%-8\% of women globally, and it is characterized by outbursts of anger, depressive mood, anxious states, affective lability, and alterations in home and work functioning [5]. Previous histories of depressive and other mood disorders have been recorded in $30 \%-70 \%$ of women with PMDD and PMS [1].

A systemic review of available evidences showed that $66.9 \%$ is the highest reported prevalence of PMDD among women, and this rate was obtained in Ethiopia [7]. In other parts of Ethiopia, the prevalence ranged from $30.3 \%$ to $39.1 \%$, which is marginally similar to the prevalence obtained in Iran (36.3\% to 59\%), Egypt (40.5\%), Nepal (38.9\% to 39.6\%), Nigeria (36.1\%), and South Korea (34.8\%) [7].

Also, studies have suggested that these symptoms occur across much of the MC and that it manifests more than twice as often in women with PMDD than in those without PMDD [4]. Additionally, women with menstrualrelated physical disorders are 1.7-3.0 times more likely (than women without menstrual-related physical disorders) to report lack of adequate and quality night-time sleep, daytime-sleepiness, nervousness, regular body aches, restlessness, sad state of mind, feelings of hopelessness and worthlessness, and that "everything was an effort" [4]. As potential indicators of depression, these symptoms are, thus, associated with significant disability and reduced quality of life.

Among studies that retrospectively diagnosed PMDD, depressive disorders (such as major and minor depression, and dysthymia) occurred in 18\%-69\% of women suffering from PMDD, but in studies that prospectively diagnosed PMDD, these disorders occurred in 12\%-25\% of this population [8]. This result is similar across nations. A survey, of 410 female university students in Nigeria, revealed that depression was the most frequent comorbid diagnosis among students suffering from PMDD [8]. Depression was present in 24\% of this population, but only in $3.6 \%$ of women without PMDD [8].

\section{Major Depressive Disorder (MDD)}

In 2001, Alonso and Coe reported that dysmenorrhea increased the predisposition of women to MDD, one of the most common mental disorders [9]. In 2005, Strine et al. reported that the rate of MDD among premenopausal women is approximately twice that of men [4].

For some women, the MC may have significant impacts on the course of current or future depressive disorders. Vulnerability towards the occurrence of depressive mood states is increased during the premenstrual phase, and existing depressive disorders may worsen [5].

In 2008, Barron et al. established that factors such as irregular MC, late menarche, and being in one's first year post-menarche are associated with MDD [1]. Barron et al. also buttressed the relationship between MDD and irregular MCs by reporting the case of a patient who experienced resolution of her treatment-resistant depressive symptoms only after the treatment of her polycystic ovarian syndrome, which restored her regular MCs [1].

In 2003, Rasgon et al. established that serum levels of estradiol are significantly lower in depressed women than in non-depressed ones, and that this might be responsible for depressed women having twice the risk of earlier peri-menopausal transition [10]. Furthermore, Rasgon et al. found that the prevalence of MDD, increases among menopausal women [10]. 
Furthermore, an association between MDD and period poverty, a state of lack of access to menstrual hygiene management products, have been established. In 2008, Haoran et al. discovered that women who suffer from period poverty every month of the year are the most likely to report moderate or severe depression, and this prevalence is followed by those who experience period poverty only in some months [5].

\section{Major Anxiety Disorders}

Independent of the impacts of race: (1) Women who have regular MCs are more than two times as likely to have an existing anxiety disorder, compared to those who have irregular MCs [1]; (2) Women with shorter cycles ( $\leq 28$ days) have 1.5 to 2 times greater risk of lifetime anxiety disorder, compared to those with normal or longer cycles [1]; and (3) There is no discernible phasic changes in the duration and severity of the anxiety disorders developed during women's MC [1].

Also, in 2001, Alonso and Coe discovered that dysmenorrhea and period poverty increase the predisposition of women to major anxiety disorders [9].

Moreover, menstrual health has its associations across four subtypes of major anxiety disorders - Generalized anxiety disorders, panic disorders, obsessive compulsive disorder, and social phobias.

Generalized Anxiety Disorder (GAD): Women suffering from GAD frequently reports premenstrual worsening [11]. Also, in 2008, Soares and Steiner recorded a high GAD prevalence of 52\% among undergoing their menstrual cycle [12]. Furthermore, the prevalence of comorbid PMDD with GAD ranges from $4 \%$ to $38 \%$, globally [11].

Panic Anxiety Disorder (PAD): Retrospective studies have found an increase in symptoms of anxiety and panic during the follicular MC phase in a considerable number of women suffering from PAD, and premenstrual exacerbations (increased severity and frequency) of these symptoms were confirmed in $50 \%$ of a sub-sample of women studied prospectively [12].

Obsessive Compulsive Disorder (OCD): Retrospective studies have found that $20 \%$ to $50 \%$ of a sample of women suffering from OCD experienced premenstrual worsening of the disease symptoms [12]. In 2005, Labad et al. also found that women, with a post-partum onset or worsening of OCD symptoms, more frequently reported premenstrual exacerbation of these symptoms and existing or previous depressive disorders, than those without these conditions [13]. This suggests that there may also be increased vulnerability of women suffering from OCD to anxiety and mood disorders during periods of abrupt variability in hormonal levels, such as the pre-menstrual and post-partum phases. Similarly, in 2006, Vulink et al. established that OCD was comorbid with PMDD in about $12 \%$ of their sample population, but this relationship only partly explained the premenstrual exacerbation of OCD among their study population [14].

Social Phobias: In 2015, SR et al. reported that social phobia is comorbid in about $20 \%$ of women suffering from PMDD, and that embarrassment due to menstruation and perceived body type and image, also increases one's predisposition to social phobias [15].

\section{Psychotic Disorders}

Studies have shown that some women develop periodic psychosis and schizophrenic symptoms during all phases of their MC [5], and that those with psychotic disorders are more vulnerable to its symptoms, which are sometimes exacerbated, during their menstrual phase [15]. A systematic review and meta-analysis, of 11 studies, investigating 742 women suffering from psychosis, showed an increased rate of psychiatric admissions in women during their menstrual phase, and that this rate is about 1.5 times more than the admission rate during other MC phases [4]. 
Strine et al. (2005) also investigated into whether psychotic symptoms, when assessed with a standardized clinician-rating scale, worsened during the follicular and luteal MC phases [4]. Four of the six studies that measured psychotic symptoms, reported exacerbation of these symptoms during these phases; and $20 \%$ to $30 \%$ of the women investigated during the two studies (that directly asked participants whether their psychosis was influenced by their MC) responded positively to this question [4]. However, the limitation of this study is that most of the reviewed/analyzed research did study women with irregular MCs. This is a cause of concern because about $40 \%$ of women suffering from psychosis do not have regular MCs [16].

\section{Bipolar Disorder (BPD)}

From a 2008 observational study of 80 women who had received treatment for BPD, Barron et al. reported menstrual disorders (amenorrhea, oligomenorrhea, polymenorrhea, and menorrhagia) in 65\% of the study population [1]. Similarly, in 2006, Joffe et al. discovered that early-onset menstrual dysfunction (within 5 years post-menarche) prior to the onset of mental disorders is more common among women suffering from BPD than among the healthy controls, and those suffering from unipolar depression [17]. This discovery suggests a relationship between BPD and abnormalities in the hypothalamic-pituitary-ovary axis [17].

Furthermore, other studies have shown that about $65 \%$ of women who are undergoing or have completed treatment for BPD reported significant mood changes across the MC, and 59\% of them, including those taking oral contraceptives, reported longer MCs [18].

Moreover, a large meta-analysis found that $44 \%-68 \%$ of the women suffering from BPD had some premenstrual-related mood changes, $25 \%-77 \%$ of these women met the criteria for premenstrual dysphoria, and $15 \%-27 \%$ of them met the criteria for PMDD [19].

\section{Borderline Personality Disorder}

A study, published in Psychological Medicine, provides preliminary evidence that women with borderline personality disorder are more likely to experience worsened symptoms of the disease during their perimenstrual phase [11]. In the study, borderline personality disorder symptoms, on average, worsened by at least 30 percent during this phase [11]. "This is equivalent to going from moderate depression to extreme depression on the rating scale", said the lead author of the paper.

\section{Sleep Disorders}

Women who suffer from severe PMS have reported poor quality of sleep, especially during their late luteal MC phase; and this decline is particularly significant when compared with the sleep quality during their early follicular phases [20].

A cross-sectional survey of female Japanese college students shown the prevalence of PMDD, PMS, and "no/mild PMS" to be $12.3 \%, 20.6 \%$, and 67.1\%, respectively [21]. Daytime-sleepiness, measured by the Japanese version of the Epworth Sleepiness Scale, was highest in the students suffering from PMDD, followed by those suffering from PMS, and then, those with "no/mild PMS" [21]. Also noteworthy is the fact that the students with "no/mild PMS" still experienced higher daytime-sleepiness than their male counterparts [20], but the underlying mechanism for this difference remains unclear.

In 2020, Miura and Honma also established that women suffering from dysmenorrhea are more prone to irregular sleep cycles that affects their general health and daily living [21].

\section{Substance Use and Abuse}

Studies have shown that women with PMS and PMDD, especially adolescents and young adults [22], are more likely to become regular users/abusers of substances (such as alcohol, tobacco and hard drugs), and these set 
of individuals are more likely to experience moderate to severe symptoms of these disorders, unlike their counterparts who use substances occasionally or have never used them [4]. The most commonly reported of these symptoms include increased tension, irregular or heavy periods, and severe dysmenorrhea [4]. Strine et al. also suggested that heavy smokers are the population of women most commonly affected by these symptoms.

Furthermore, reports have shown that overweight and obesity, which are commonly associated with PMS and PMDD, predisposes women to substance use and abuse [4]. Additionally, it has been reported that women with shorter cycles ( $\leq 28$ days) have 1.5 to 2 times more risk of lifetime substance use, abuse, and dependence disorders [1].

\section{Suicidal Ideation and Attempt}

In 2015, Zengin et al. reported that the majority of suicide attempts by women occurred during their menstrual phase when the levels of estrogen and progesterone are at their lowest [23]. In 2019, Jang and Elfenbein recorded a $26 \%, 17 \%$ and $20 \%$ prevalence of increased risk of suicide deaths, suicide attempts, and psychiatric admissions, respectively, among women during their MC [24]. The study also recorded a 13\% prevalence of higher risk of psychiatric admissions during the premenstrual MC phase of the sample population [24]. Also, in 2014, Klatzkin et al. reported that the prevalence of suicidal ideation among pre-menopausal women is unrelated to their MC phase [2].

In 2008, Yonkers et al. reported that among 426 women in six outpatient obstetrics clinics, who suffered from menstrual-related mental disorders, $24 \%$ of them reported suicidal ideation, occurring during all days, multiple days, and more than half the period of their menstrual phase [25].

\section{Positive Associations}

Although studies into the link between mental and menstrual health have focused almost entirely on the negative associations between these entities, there is evidence that $5-15 \%$ of women have positive mental experience(s) during their MC. In 2012, Romans et al. reported that $66 \%$ of patients attending a gynecologic clinic for non-menstrual-related matters reported one or more positive mental experience(s) during their MCs [26].

\section{Bodily Mechanisms Implicated in the Links Between Menstrual and Mental Health}

Sex steroids, which crosses the blood-brain barrier and are also be synthesized within the brain, affect one's mental state by mediating changes in the neurotransmitter systems of different brain regions, mostly by enhancing the activities of neuroamines such as noradrenaline, dopamine, serotonin, and g-aminobutyric acid [12]. The central gray-matter, amygdala, hippocampus, basal ganglia, and various regions of the cortex, which are parts of the brain implicated in anxiety disorders, contain receptors of female sex steroids [12]. Additionally, CNS activities of neuroamines, which are also implicated in the pathophysiology of anxiety disorders, are altered by these steroids as well [12].

Overall, estrogens, especially estradiol, are agonists of serotonin and noradrenaline activities-Estrogen increases the synthesis and release, inhibits the reuptake, and slows down the degradation of serotonin and noradrenaline [12]. Also, the psychological distress, that occurs regularly during the luteal MC phase, especially of women with serotonin dysregulation, is perhaps related to the population's altered sensitivity to progesterone and its metabolites (e.g. allopregnanolone), which alters neuroamine concentrations [4]. 
Furthermore, both PMDD and PMS have been linked to serotonin dysregulation [8]. In women suffering from PMDD, levels of serum serotonin and its platelet uptake are decreased, while in those with PMS, their symptoms are exacerbated by the depletion of tryptophan, the precursor to serotonin [8].

Additionally, in animal models, serotonin inhibits the expression of the aggression and irritability-predominant symptoms of PMS [8]. Fluoxetine, for instance, reduces the aggressive behaviors promoted by the activities of estrogen and progesterone in ovariectomized rats [8]. These animal-based experiments reflect a relationship between serotonergic-transmission and cycle-dependent behaviors that are related to female sex hormones [8].

Moreover, the major evidence that associates PMDD and MDD with serotonin-dysregulation is the high efficacy of serotonergic anti-depressive drugs [8]. Unlike, serotonergic antidepressants, non-serotonergic ones do not relieve PMDD, but sometimes, it relieves MDD. Whether women who respond to non-serotonergic antidepressive drugs have lesser risk of suffering from PMS is not known, but it is of great interest.

Furthermore, the pathophysiological pathway(s) that lead some women to suffer from premenstrual worsening of GAD, are not known. However, some studies, albeit limited, have suggested that GAD is associated with decreased levels of serum pregnanolone sulfate [12]. But alterations in the levels of neuro-steroids in women who suffer from premenstrual exacerbation of GAD have not yet been investigated [12].

In addition, available evidence highlights the MC-related influences of the female sex hormones on the course of OCD in some women. These influences perhaps occur via the agonistic and antagonist effects of serotonin on dopamine neurotransmission, causing increased dopamine activities that, in turn, results in OCD exacerbation [12].

Also, several authors have suggested that the rise in concentration of serum progesterone during women's luteal MC phase could be the cause of their increased daytime-sleepiness during this phase [20]. This theory further suggests that the levels of daytime-sleepiness in women during their follicular MC phase, is perhaps similar to the levels in men [20].

Other reported contributors to menstrual-related mental disorders include genetic susceptibility, putative brain structural and functional differences, altered sensitivity to brain-derived neurotrophic factor, and further involvement of the hypothalamic-pituitary-adrenal and hypothalamic-pituitary-gonadal axes in trauma, resilience, and inflammation $[4,8,12]$.

\section{Research and Treatment Gaps}

Although anecdotal evidence highlights the associations between mental and menstrual health, very limited research has investigated the relationships between specific mental disorders and comorbid menstrual disorders, despite the results' prospects in guiding the provision of adequate and quality healthcare to women [1].

Furthermore, there have not been adequate verification of findings of research into the links between mental and menstrual health. There are still wide result variations across studies. For example, of 47 studies into this subject matter, $38.3 \%$ found no association between mood and MC; and only $14.9 \%$ established a relationship between negative mood and women's premenstrual phase [26]. Moreover, in the latter studies, a commonly observed pattern was for the women's negative mood states to persist throughout their menstrual phases and not be restricted to their pre-menstrual phases alone [26].

In addition, many psychiatry research instruments investigate only negative experiences or place more emphasis on negative than positive experiences [26]. This impedes a complete description of the effects of menstrual health on mental health, and vice versa. This should be remedied urgently due to the fact that women 
may generically have greater amplitude for both positive and negative mood states changes, in certain $\mathrm{MC}$ phases. Moreover, if only negative mood changes are investigated, the studies would, falsely, conclude that the negative axis is the only direction of change.

In the last 50 years, PMS has become as a well-recognized disorder, for which many efficacious treatments are available. Unfortunately, it has also become the target of numerous jokes among the general public. Moreover, because of the widespread public awareness of negative premenstrual experiences, the phrase "PMS" has found its application in popular vernacular languages as a noun, adjective and verb (e.g. "I'm PMS ing”) [1]. This reality has spurred many individuals and organizations, who seek to generate income at all cost, to market over-the-counter remedies as a "sure cure" for this common disease, thus exploiting the fact that "many women believe they suffer from PMS" [1]. Therefore, there is an urgent need to sensitize the general public of the differences between the usual premenstrual experience of ovulatory women (wherein PMS symptoms are mild, or premenstrual molimina signals impending menstruation) from PMDD (wherein symptoms, particularly the psychological ones, lead to major distress that can disrupt daily living and interpersonal relationships). Another major task for the medical profession is to offer appropriate and timely interventions to these disorders.

\section{Conclusion}

A broader understanding of the highlighted links between mental health and MCs is crucial to the provision of adequate and quality healthcare for women globally.

Also, women with mild menstrual-related mental disorders should receive counsel about minor lifestyle changes that may help combat these disorders. While those with moderate, severe, and extremely severe ones should receive early and adequate interventions, with or without medications.

\section{Acknowledgement}

The author wishes to acknowledge all core team members of The TriHealthon for their contribution towards the development of this paper.

\section{Conflict of interest}

The author declare no conflict of interest.

\section{Funding}

The author received no external funding for the development of this paper. 


\section{Reference}

1. Barron ML, Flick LH, Cook CA, et al. (2008) Associations between psychiatric disorders and menstrual cycle characteristics. Archives of psychiatric nursing, 22(5), 254-265. DOI: 10.1016/j.apnu.2007.11.001

2. Klatzkin RR, Bunevicius A, Forneris CA, et al. (2014) Menstrual mood disorders are associated with blunted sympathetic reactivity to stress. Journal of psychosomatic research, 76(1), 46-55. DOI: 10.1016/j.jpsychores.2013.11.002

3. Yu M, Han K, \& Nam GE. (2017) The association between mental health problems and menstrual cycle irregularity among adolescent Korean girls. Journal of affective disorders, 210, 43-48. DOI: 10.1016/j.jad.2016.11.036

4. Strine TW, Chapman DP, \& Ahluwalia IB. (2005) Menstrual-related problems and psychological distress among women in the United States. Journal of women's health (2002), 14(4), 316-323. DOI: 10.1089/jwh.2005.14.316

5. Haoran Z, Ning LX, Boddu S. (2018) Effects of Premenstrual Syndrome Related Psychiatric Disorder on Physical and Mental Health Status of Adolescents- A Short Review. Int J Womens Health Wellness, 4:083. DOI: $10.23937 / 2474-1353 / 1510083$

6. Matsumoto T, Egawa M, Kimura T, et al. (2019) A potential relation between premenstrual symptoms and subjective perception of health and stress among college students: a cross-sectional study. BioPsychoSocial Med 13, 26. DOI: 10.1186/s13030-019-0167-y

7. Tsegaye D, \& Getachew Y. (2019) Premenstrual dysphoric disorder and associated factors among female health science students in Wollo University, Ethiopia, 2017/18. matern health, neonatol and perinatol 5, 8. DOI: 10.1186/s40748-019-0102-z

8. Kim DR, \& Freeman EW. (2010) Premenstrual Dysphoric Disorder and Psychiatric Comorbidity. Psychiatric Times, 27(4). Available from https://www.psychiatrictimes.com/view/premenstrualdysphoric-disorder-and-psychiatric-comorbidity

9. Alonso C, \& Coe CL. (2001) Disruptions of social relationships accentuate the association between emotional distress and menstrual pain in young women. Health psychology: official journal of the Division of Health Psychology, American Psychological Association, 20(6), 411-416.

10. Rasgon N, Bauer M, Glenn T, et al. (2003) Menstrual cycle related mood changes in women with bipolar disorder. Bipolar disorders, 5(1), 48-52. DOI: 10.1034/j.1399-5618.2003.00010.x

11. University of Illinois at Chicago. (2018) Symptoms worsen around menses for people with borderline personality disorder. Available at https://www.sciencedaily.com/releases/2018/05/180531143106.htm

12. Soares CN, \& Steiner M. (2008) The Complex Interrelationships of Menstrual Cyclicity and Anxiety Disorders. Psychiatric Times, Psychiatric Times, 25 (5). Available from https://www.psychiatrictimes.com/view/complex-interrelationships-menstrual-cyclicity-and-anxietydisorders

13. Labad J, Menchón JM, Alonso P, et al. (2005) Female reproductive cycle and obsessive-compulsive disorder. The Journal of clinical psychiatry, 66(4), 428-546. DOI: 10.4088/jcp.v66n0404

14. Vulink NC, Denys D, Bus L, et al. (2006) Female hormones affect symptom severity in obsessivecompulsive disorder. International clinical psychopharmacology, 21(3), 171-175. DOI: 10.1097/01.yic.0000199454.62423.99 
15. SR Q, VB W, AD G, et al. (2015) Are Menstrual Problems Associated with the Mental Health? A Cross Sectional Study among the Graduation College Girls. IIUM Medical Journal Malaysia, 14(2). DOI: 10.31436/imjm.v14i2.435

16. Reilly T. (2019). Some women with psychotic disorders are more vulnerable around the time of menstruation, our analysis suggests. The Conversation. Available from https://theconversation.com/some-women-with-psychotic-disorders-are-more-vulnerable-around-thetime-of-menstruation-our-analysis-suggests-114935

17. Joffe H, Kim DR, Foris JM, et al. (2006) Menstrual dysfunction prior to onset of psychiatric illness is reported more commonly by women with bipolar disorder than by women with unipolar depression and healthy controls. The Journal of clinical psychiatry, 67(2), 297-304. DOI: 10.4088/jcp.v67n0218

18. Cardoso, L.F., Scolese, A.M., Hamidaddin, A. et al., 2021. Period poverty and mental health implications among college-aged women in the United States. BMC Women's Health 21, 14. DOI: $10.1186 / \mathrm{s} 12905-020-01149-5$

19. Purse M. (2020) Effects of Premenstrual Symptoms on Bipolar. Available from https://www.verywellmind.com/effects-of-premenstrual-symptoms-on-bipolar-379963

20. Komada Y. (2020) Relationship of women's reproductive health and menstrual problems with sleep and circadian rhythm. Sleep Biol. Rhythms 18, 1. DOI: 10.1007/s41105-019-00247-8

21. Miura J, \& Honma R. (2020) Daytime sleepiness in relation to gender and premenstrual symptoms in a sample of Japanese college students. Sleep Biol. Rhythms 18, 3-8. DOI: 10.1007/s41105-019-00236$\mathrm{X}$

22. Bertone-Johnson ER, Hankinson SE, Johnson SR, et al. (2008) Cigarette smoking and the development of premenstrual syndrome. American journal of epidemiology, 168(8), 938-945. DOI: 10.1093/aje/kwn194

23. Zengin Y, Calik M, Buyukcam F, et al. (2015) 'The relationship between suicide attempts and menstrual cycles in the emergency department and the sociodemographic and clinical characteristics of these patients', Eurasian Journal of Emergency Medicine, 14(3), 118+. DX.DOI: 10.5152\%2Fjaem.2015.91069

24. Jang D, \& Elfenbein HA. (2019) Menstrual Cycle Effects on Mental Health Outcomes: A MetaAnalysis. Archives of suicide research : official journal of the International Academy for Suicide Research, 23(2), 312-332. DOI: 10.1080/13811118.2018.1430638

25. Yonkers KA, O'Brien PM, \& Eriksson E. (2008) Premenstrual syndrome. Lancet (London, England), 371(9619), 1200-1210. DOI:10.1016/S0140-6736(08)60527-9

26. Romans S, Clarkson R, Einstein G, et al. (2012) Mood and the menstrual cycle: a review of prospective data studies. Gender medicine, 9(5), 361-384. DOI: 10.1016/j.genm.2012.07.003 